\title{
Factors Affecting the Use of Maternal and Child Health Services in Eritrea
}

\author{
Gebremichael Kibreab Habtom* \\ University of Asmara, Eritrea
}

Submission: March 07, 2017; Published: May 31, 2017

*Corresponding author: Gebremichael Kibreab Habtom, College of Business and Economics, University of Asmara, Eritrea, Tel. 291-1-7146534; Fax: 291-1-126422; Email: gmichaelkibreab@gmail.com

\section{Abstract}

Background: The study aims to determine the factors that affect the use of maternal and child health services in Eritrea. It analyses the use of antenatal, postnatal, and delivery care services in Eritrea.

Methods: The data for this study was taken from the Eritrea Population and Health Survey conducted on the year 2010 by the National Statistics Office. To substantiate the EPHS 2010 data an interview was conducted in 2016 (May-June) with selected key informants (health professionals, traditional birth attendants and women who have children below 5 years old and women three month pregnant preceding the study). About 110 persons were interviewed in Zoba Debub and Zoba Maekel of Eritrea. A combination of descriptive and logistic regression analysis was employed to the gathered data to understand the prevailing situation and to assess the differentials in utilization of maternal care services.

Results: This study shows that the most important factors influencing the use of maternal and child health services in Eritrea are demographic and socio cultural in nature. It was found that women delivering at younger age were more likely to use antenatal care, receive skilled attendance at delivery and use postnatal care. Furthermore woman who goes for antenatal care is more likely to deliver in a health facility or deliver at home with professional help than a woman who does not go for antenatal check-ups. Women from families living at the lowest wealth quintile were also less likely to use maternal health services in comparison with women living in families in the highest wealth quintile. The three indicators of the use of maternal health services increased sharply with increased levels of education of mother. The gender of the health staff appears also to influence the use of maternal health services. The analysis further indicated that women with less education in the lowest wealth quintile and women who live in the rural areas are continuing to use traditional medicine for maternal and child health services in Eritrea.

Conclusion: Socio-cultural and demographic factors are the most important variables that affect the use of maternal and child health services in Eritrea. Among socio-cultural factors traditional medicine continue to play an important role in maternal and child health services in Eritrea. Most rural communities in Eritrea use traditional medicinal herbs and other local ingredients to treat both diseases and common sicknesses. This indicated that effective health agenda for Eritrean can never be achieved by modern biomedicine alone unless it is complemented by traditional medical practice. The most workable health agenda for Eritrea is the institutionalization of traditional medicine in parallel (not in complete fusion) with modern medicine, within the national health care scheme in order to move the health agenda forward. The Ministry of Health, therefore, should use such findings as an insight for the modification of plans and policies for future development of maternal and child care services in Eritrea.

Keywords: Eritrea; Maternal and child health; antenatal care; Postnatal care; Delivery care; Traditional medicine

\section{Introduction}

Millions of women in developing countries experience life threatening and other serious health problems related to pregnancy or childbirth. Complications of pregnancy and childbirth cause more deaths and disability than any other reproductive health problems [1]. The World Health Organization (WHO) estimates that 58,000 women of reproductive age die each year from complications arising from pregnancy, and a high proportion of these deaths occur in Sub-Saharan Africa. The ratio of maternal mortality in the region is one of the highest in the world, reaching levels of 686 per 100,000 live births [2,3].
Health is an integral part of the national development program in Eritrea. Eritrea is an East African country, which is bordered with Ethiopia in the south, with Sudan in the north and the west, with Djibouti in the southeast, and with the Red Sea in the east. Eritrea got independence from Ethiopia in 1993 after 30 years of armed struggle. Eritrea's population is estimated to be 5.2 million, with an average life expectancy of 60.4 years and a literacy rate of 67.5 percent [4]. The population pyramid follows an early development pattern: $43 \%$ of the population is under 15 years old, $6 \%$ above 65 years old and nearly $70 \%$ of the 
population is composed of children and women in child-bearing age $[5,6]$. About $52 \%$ of the Eritrean population is under 18 years old and $17 \%$ are under five years old [7]. $80 \%$ of the people live in rural areas, and the population density ranges from 36.3 to 40.6 people per sq. $\mathrm{km}$ [8]. Agriculture is the main stay of the Eritrean economy whereby $80 \%$ of the population depends for its livelihood. About 30\% of Eritreans are semi-nomadic or agro-pastoralists. For the year 2010, the GDP for Eritrea was estimated at US $\$ 3.625$ billion together with a per capita estimate of US\$681 [9].

In its poverty reduction strategy (2001-2002) and macropolicy (1994) the government of Eritrea clearly stated that health is the key input for national development. To this end, over the last 21 years the scope and range of tasks and responsibilities of the government has increased tremendously, thus resulting in a significant increase in health care facilities. For instance, the number of hospitals increased from 16 in 1990 to 28, health centers from 4 to 63 , health stations from 72 to 249 , clinics from 34 to 74 , and pharmacies from 15 to 32 [3]. Overall, the total number of health facilities increased from 126 in 1991 to 340 in 2010, an increase of 170 percent. The number of nurses and physicians also increased significantly in the same period.

However, despite tremendous increase in health care facilities and health care personnel, the health status of many Eritreans remains poor. Preventable diseases are still responsible for about 70 percent of the burden of diseases in Eritrea. Communicable and nutritional deficiency diseases such as diarrhea, acute respiratory infections, tuberculosis, malaria, HIV/AIDS, skin and parasitic infections are the major health problems facing the poor in Eritrea. In 2002 these diseases were responsible for about $60 \%$ of outpatient and $40 \%$ of inpatient incidences and $56 \%$ of inpatient deaths [10]. The utilization of maternal health services (ante-natal, delivery and postnatal) and family planning services are very low in Eritrea. For instance, in 2002 only 18 percent of pregnant women were registered for antenatal care in their first trimester, 56 percent in the second trimester, and 26 percent in their third trimester [10]. Eritrea Demographic and Health Survey (EDHS) 2002 also reveal a similar result that is only 22 percent of all the respondents were registered for antenatal care in their first trimester. Delivery services have also low rate of utilization, only 34 percent of all deliveries are attended by health professionals. The utilization of family planning services is the lowest of all health related services in Eritrea. Contraceptive prevalence rate among currently married women is 8.0 percent, and among all women is 5.8 percent [11]. The maternal mortality rate in 1995 was estimated to be 998 per 100,000 live births [12].

One explanation for poor health outcomes among women and children is the nonuse of modern health care services by a sizable proportion of women in Eritrea. Studies demonstrating the high level of maternal mortality and morbidity in developing countries and research identifying causes of maternal deaths have repeatedly emphasized the need for antenatal care and availability of trained personnel to attend women during labor and delivery [13,14]. A common error in many health care policies in developing countries is that the implicit assumption that outcomes and health usage can simply be achieved by the provision of medical care through the construction of clinics, the training of nurses, the provision of drugs, etc. This is only the supply side of the problem. High quality health care provision is only translated into a better health of the population if individuals and households use of the health care system. Illness recognition and hence considering health care is still an important problem in Eritrea. Health care facilities are not used by all who need it. The purpose of this study is, therefore, to understand the current status of utilization of maternal health services (antenatal, delivery and postnatal) in Eritrea by elucidating the various factors influencing the use of these services in the country. It is hoped that the result of the study will improve policy makers' understanding of the determinants of maternal and child mortality and morbidity in the country and serves as an important tool for any possible intervention aimed at improving the low utilization of maternity care services in the country.

Despite the fact that maternal health care utilization is essential for further improvement of maternal and child health little is known about the current magnitude of use and factors influencing the use of these services in Eritrea. The utilization of maternal health services is a complex phenomenon and it is influenced by several factors. The factors affecting the use of these services, therefore, need to be clearly understood. Hence, we forward the following question to assess the variables that affect the use of maternal and child health services in Eritrea: what are the factors that affect the use of maternal and child health services in Eritrea?

\section{Review of Related Literature}

The use of maternal and child health services is a complex behavioral phenomenon. It is related to the organization of the health-delivery system and is affected by the availability, quality, costs, continuity and comprehensiveness of services; social structure and health beliefs also affect use $[15,16]$. For preventive services like prenatal care, family planning orimmunizations, the perception of need is more complicated than in the case of disease recognition, and involves beliefs about susceptibility, consequences and effectiveness of the intervention [17]. Kroeger [16], Anderson and Newman [18], Newbold et al. [19], Henderson et al. [20], and Fosu [21] have shown that the use of maternal and child health services is a function of three sets of individual characteristics:

A. Predisposing characteristics, i.e. age, household size, education, number of previous pregnancies, health related attitude; 
B. Enabling characteristics, i.e. income, characteristics of health care system and access, and availability of health facilities; and

C. Need characteristics, i.e. characteristics of illness, perceived health status, and expected benefit from treatments.

Fosu [21] argued that the predisposing factors reflect the fact that families with different characteristics have a different propensity to use health services, while the enabling factors reflect the fact that some families, even if predispose to use health services, must have some means to obtain them, i.e. income, access and availability of health services. According to Anderson \& Newman [18] the need factor reflects the perceived health status, as indicated by severity of the morbidity conditions or the number of morbidities. The presence of predisposing and enabling components may not be enough for a mother to seek health care. She must perceive the disease as serious and believe that the treatment will provide the expected benefits [21]. If a woman ever had a still birth in a previous pregnancy, the use of maternal care services would be higher because of known risk factor [22]. Need represents the most immediate cause of health service use. The need for health care can be measured in a variety of ways: self-perceived health status, number of morbidity symptoms, or duration and severity of disability [15].

A review of the literature suggests that in developing countries like Eritrea, the use of modern healthcare such as maternal health services can be influenced by the sociodemographic characteristics of women, the cultural context, and the accessibility of these services. A number of sociodemographic characteristics of the individual hence affect the under lying tendency to seek care [23]. For instance, women's current age plays an important role in the utilization of medical services $[15,21,24]$. Women's age may sometimes serve as a proxy for the women's accumulated knowledge of health care services, which may have a positive influence on the use of health service. On the other hand, because of development of modern medicine and improvement in educational opportunities for women in recent years, younger women might have an enhanced knowledge of modern health care services and place more value upon modern medicine. Several studies also showed a strong association between birth order and use of health care services $[24,25]$.

Because of perceived risk associated with first pregnancy, a woman is more likely to seek maternal health care services for first order than higher order births. Having more children may also cause resource constraints, which have a negative effect on health care utilization [25]. Women with a large number of children underutilize available health services because of too many demands on their time force them to forgo health care [26]. Hence, the greater confidence and experience of the older and higher parity women, together with greater responsibilities within the household and for child care, have been suggested as explanatory factors for their tendency to use services less frequently [27]. Other studies showed women's education as an important variable in the use of modern health care services $[21,24,28,29]$. Women in higher education groups tend to exhibit patterns of more frequent use of maternal health services than women in the lower education groups [23,30-32].

Another important factor in the utilization of maternity care services, especially in Africa, is the cultural background of the woman $[30,33]$. The cultural perspective on the use of maternal health services suggests that medical need is determined not only by the presence of physical disease but also by cultural perception of illness [23]. In most African rural communities, maternal health services coexist with indigenous health care services; therefore, women must choose between the options [23]. The use of modern health services in such a context is often influenced by individual perceptions of the efficacy of modern health services and the religious beliefs of individual women [34]. Moreover, in many parts of Africa, women's decision- making power is extremely limited, particularly in matters of reproduction and sexuality. In this regard, decisions about maternal care are often made by husbands or other family members [35]. Availability of women's time is also important. In developing countries, women spend more time on their multiple responsibilities for care of children, collecting water or fuel, cooking, cleaning, growing food, and trade than on their own health [2].

Several other studies also found that physical proximity and accessibility of health services has been shown to be an important determinant of utilization of health services in developing countries [36-39]. In most rural areas in Africa, one in three women lives more than five kilometers from the nearest health facility [2]. The scarcity of vehicles, especially in remote areas, and poor road conditions can make it extremely difficult for women to reach even relatively nearby facilities. Walking is the primary mode of transportation, even for women in labor $[2,40]$. In rural Tanzania, for example, 84 percent of women who gave birth at home intended to deliver at a health facility but did not due to distance and lack of transportation [41]. Furthermore, fees reduce women's use of maternal health services and keep millions of women from having hospital-based deliveries or from seeking care even when complications arise. Even if formal fees are low or nonexistent, there may be informal fees or other costs that pose significant barriers to women's use of services. These may include costs of transportation, drugs, food, or lodging for the woman or for family members who help care for her in the hospital $[42,43]$.

In Eritrea, studies addressing the factors influencing the utilization of maternity care services are scant. The few studies that do exist focused predominantly on urban areas and have identified some important determinants of use of maternity care services in the country. Habtomand Ruys [44] in their study of the choice health care provider in Eritrea found that women who did not receive modern health care were often poor and illiterate, 
with limited knowledge of maternity care services. Another study in Eritrea showed that lack of time, absence of illness, and lack of awareness are the major reasons for nonattendance for antenatal care [45]. Socio-demographic factors including parity, age, and education appeared to influence the use of maternity care services in urban areas [45]. In contrast, distance and travel time were identified as important factors in the rural parts of Eritrea [45].

\section{Data and Method of Analysis}

The data for this study come from the 2010 Eritrea Population and Health Survey (EPHS), which was conduct for the third time in the country. The data used for this analysis are unique in terms of content, geographic coverage, and timeliness compared with previous studies on the use of maternal health services in the country. The survey collected information from a nationally representative sample of 36,000 women age 15-49. This study analyzes responses from 13,817 women age 15-49, who have at least one child under age five at the time the survey was fielded. To substantiate the EPHS 2010 data an interview was conducted in 2016 (May-June) with selected key informants (health professionals, traditional birth attendants and women who have children below 5 years old and women three month pregnant preceding the study). About 110 persons were interviewed in Zoba Debub and Zoba Maekel of Eritrea. The interviews were conducted by senior public administration students. As part of their senior research project the students were advised to conduct research on the use of maternal and child health services in Eritrea. A number of specific questions were asked to women about their most recent pregnancy and live birth in the five years preceding the survey. Women were asked 1) whether they were checked by a trained health professional, that is, doctor, nurse, or midwife, at least once during pregnancy, i.e., antenatal care (ANC); 2) whether they were attended by a trained health professional during their delivery, i.e., professionally assisted delivery (PAD); and 3) for those mothers who delivered outside a health facility, whether they received a medical checkup from a health professional from 3-41 days after delivery, i.e., postnatal care (PNC).

The study aims to determine the factors that affect the utilization of pre and postnatal care, including the place of delivery. Each of the independent variables was selected for inclusion in the analysis based on previous literature. Independent variables included in the present study were maternal age at birth, parity, number of living children, educational status of women, marital status, work status, residence and socioeconomic status (wealth quintile).

\section{Methods of analysis}

The unit of analysis for this study was women who have children below 5 years old and women three month pregnant preceding the study. A combination of descriptive and logistic regression analysis was employed to the gathered data to understand the prevailing situation and to assess the differentials in utilization of maternal care services. A multivariate analyses was carried out for the three maternal health care variables .For the purposes of such analysis, the response category was collapsed to create a dichotomous variable on the basis of whether or not the woman had received maternal health care. Since the interest is in identifying women at risk because they did not receive care, the outcome variable was coded as 1 if the woman received antenatal care and as 0 if she did not receive antenatal care. The same coding procedure was applied for delivery and postnatal care.

Logistic regression was used for the multivariate analysis. The logistic model considers the relationship between a binary dependent variables and a set of independent variables. The logistic model for $\mathrm{K}$ independent variables $(\mathrm{X} 1, \mathrm{X} 2, \mathrm{X} 3, \ldots . . \mathrm{Xk})$ is given as:

\section{$\operatorname{Logit} \mathrm{P}(\mathrm{x})=\alpha+\Sigma \beta \mathrm{iXi}$}

$\operatorname{Exp}(\beta i)=$ odds ratio for a person having characteristic $\mathrm{i}$ versus not having characteristic $i$.

\section{$\beta=$ Regression coefficient $\alpha=$ Constant}

Confidence intervals (CIs) and P-values were presented for the odds ratios. CI gives a lot of information. For instance, a CI covering 1 implies that there is no effect of the factor under consideration. Otherwise, there is an effect of that variable.

\section{Results}

\section{Characteristics of respondents}

The age at which childbearing starts has important consequences for the overall level of fertility as well as the health and welfare of the mother and the child. As indicated in (Table 1) below, early childbearing is not common in Eritrea with the majority of women becoming mothers after age 20 . About $68 \%$ of women age $20-34,21 \%$ of women age 35 and above, and $11 \%$ of women age less than 20 years had given birth to their first child before age 15 . The median age at first birth among women age 25-49 is 22 years [45]. Information on children ever born is useful for observing how average family size varies across age groups and for observing the level of primary infertility. Comparison of the differences in the mean number of children ever born and surviving reflects the cumulative effects of mortality levels during the period in which women have been bearing children. On average, Eritrean women age 15-49 have given birth to 2.6 children, of which 2.4 children are still alive, indicating that $8 \%$ of the children ever born have died [45]. The mean number of children ever born has declined from 3.0 children in 1995 to 2.7 in 2002 and to 2.6 in 2010 [45]. About $25 \%$ of the respondents have 1-2 living children, $34 \%$ have no children, $20 \%$ have $3-4$ living children and about $21 \%$ have 5 and above living children. The number of children that women have borne increases with age, from 0.1 children for women age 15-19 to more than two children for women in their late twenties, about five children for women in their late thirties, and to nearly six children for women at the end of their reproductive years [45-49]. 
Table 1: Characteristics of respondents.

\begin{tabular}{|c|c|c|c|c|c|}
\hline aVariables & Percentage & Number & Variables & Percentage & Number \\
\hline $\begin{array}{c}\text { Mother's age at last } \\
\text { birth } \\
\text { (years) }\end{array}$ & & & $\begin{array}{l}\text { Administrative } \\
\text { region (Zoba) }\end{array}$ & & \\
\hline$=>35$ & 21.11 & 2,917 & Debubawi Keih Bahri & 1.51 & 209 \\
\hline $20-34$ & 67.71 & 9,356 & Maekel & 18.33 & 2,533 \\
\hline$<20$ & 11.17 & 1,544 & Semenawi Keih Bahri & 11.81 & 1,632 \\
\hline Birth order & & & Anseba & 15.44 & 2,133 \\
\hline \multicolumn{6}{|l|}{6} \\
\hline+ & 23.25 & 3,213 & Gash-Barka & 24.93 & 3,445 \\
\hline $4-5$ & 23.82 & 3,292 & Debub & 27.97 & 3,864 \\
\hline $2-3$ & 34.43 & 4,758 & Mother's education & & \\
\hline 1 & 18.48 & 2,554 & No education & 45.13 & 6,234 \\
\hline Residence & & & Primary & 24.82 & 3,429 \\
\hline Rural & 67.81 & 9,369 & Middle & 16.61 & 2,295 \\
\hline Asmara & 11.87 & 1,640 & Secondary or above & 13.44 & 1,856 \\
\hline Other urban & 20.32 & 2,808 & $\begin{array}{c}\text { Current marital } \\
\text { status }\end{array}$ & & \\
\hline Wealth quintile & & & Never married & 28.11 & 2,878 \\
\hline Lowest & 18.22 & 2,518 & $\begin{array}{l}\text { Married/Living } \\
\text { together }\end{array}$ & 60.39 & 6,183 \\
\hline
\end{tabular}

Marriage is one of the main indications of the exposure of women to the possibility of pregnancy and hence is important to the understanding of fertility. Early age at first marriage in a population is usually associated with a longer period of exposure to the risk of pregnancy and thus higher fertility levels. The early initiation of childbearing associated with early marriage may also adversely affect women's and children's health. The proportion of women age 15-49 that have never married (or lived with a man) is $28 \%$, showing a $5 \%$ increment from EDHS 2002, which was $23 \%$. The largest proportion of never married women is observed in the age group 15-19, of whom $81 \%$ had never been married in 2010 [45]. This proportion was 62\% in EDHS 1995 and $69 \%$ in EDHS 2002. In this study women, who are currently married account for $60 \%$, never married $28 \%$ and divorced/ separated/widowed $11 \%$. About $25 \%$ of the respondents have 1-2 living children and 35\% have no living children.

Information on household assets was used to create an index that is used throughout this report to represent the wealth of the households interviewed in the Eritrea Population and Health Survey (EPHS) 2010. The wealth index was developed and tested in a large number of countries in relation to inequalities in household income, use of health services, and health outcome [46]. It has been shown to be consistent with expenditure and income measures [46]. The wealth index is constructed using household asset data, including ownership of consumer items ranging from a television to a bicycle or car, as well as dwelling characteristics such as source of drinking water, sanitation facilities, and type of flooring material. The resulting wealth index has a mean of zero and a standard deviation of one. Once the index is computed, national-level wealth quintiles (from lowest to highest) are formed by assigning the household score to each de-jure household member, ranking each person in the population by that score, and then dividing the ranking into five equal categories, each comprising $20 \%$ of the population [45]. Throughout this study quintiles are expressed in terms of quintiles of individuals in the overall population rather than quintiles of individuals at risk for any one health or population indicator. Urban population is represented in the fourth and highest quintiles (86\%), while about 6 in 10 households in rural areas are in the lowest and second wealth quintiles [45]. The wealth quintile distribution among administrative regions also shows large variations. The three administrative regions with lowest wealth quintile are Anseba 33\%, Gash-Barka 31\%, and Semenawi Keih Bahri 26\%. In contrast, Maekel has the largest proportions in the highest wealth quintile, 64\%. Furthermore, the EPHS 2010 shows that about $77 \%$ of women aged $15-49$ in the sample were not currently employed, only $23 \%$ were employed.

In the EPHS 2010, information on educational attainment was collected for women aged $15-49$ years. About $45 \%$ of women were with no educated, $25 \%$ with primary education, $17 \%$ with middle level education, and $13 \%$ were with secondary or above education. Educational attainment is much higher among the urban population than among the rural population. In urban areas, $25 \%$ of females and $15 \%$ of males have no education, compared with $58 \%$ of females and $46 \%$ of males in rural areas [45]. Among administrative regions, the proportion of females and males with no education is highest in Debubawi Keih Bahri 


\section{Journal of Complementary Medicine \& Alternative Healthcare}

(72 and 52 percent, respectively) and lowest in Maekel (21 and 10 percent, respectively) [45].

\section{Multivariate analysis}

The results of this study showed that $88.5 \%$ of the respondents used antenatal services at least once during their most recent pregnancy. The percentage of mothers whose last delivery was assisted by skilled personnel was 34\% whereas only $13.3 \%$ women received postnatal care within two weeks after their most recent delivery. The results of the multivariate analysis for the use of antenatal, delivery and postnatal care are presented in detail in sections 4.2.1-4.2.3.

Antenatal care: Antenatal care (ANC) is provided to enable healthy motherhood through early detection of risk factors and to take timely intervention when necessary. One of the major goals of antenatal care is to identify and treat problems that may occur during pregnancy such as anemia, high blood pressure, and genital infections. In the 2010 EPHS, information on ANC coverage was obtained from women who had a birth in the five years preceding the survey. For women with two or more live births during the five year period, data refer to the most recent birth only.

Eighty-nine percent of women who had a live birth in the five years before the survey attended antenatal care for the most recent birth. ANC coverage by a skilled provider increased from 48 percent in 1995 to 89 percent in 2010 [45]. The increase in antennal care has occurred in all subgroups. It is encouraging to note that there has been an increase in antenatal care coverage in all administrative regions, and among women with no education. The overall increase in antenatal care in the country is due to almost entirely to a tremendous increase in antenatal care capacity and coverage for all, including uneducated women.

Table 2: Results of the multivariate analysis of the variables related to the use of maternal and child health services in Eritrea.

\begin{tabular}{|c|c|c|c|c|c|c|c|}
\hline & \multirow[t]{2}{*}{$\mathbf{N}$} & \multicolumn{2}{|c|}{ Received any ANC } & \multicolumn{2}{|c|}{ Skilled attendance at delivery } & \multirow[b]{2}{*}{$\%$} & \multirow{2}{*}{$\begin{array}{c}\text { Received PNC } 3 \text { - } 41 \text { days } \\
\text { OR }(95 \% \mathrm{CI})\end{array}$} \\
\hline & & $\%$ & OR $(95 \%$ CI $)$ & $\%$ & OR $(95 \% \mathrm{CI})$ & & \\
\hline All respondents & 13,817 & 88.5 & & 34.1 & & 13.3 & \\
\hline \multicolumn{8}{|c|}{ Individual variables } \\
\hline \multicolumn{8}{|c|}{ Mother's age at last birth } \\
\hline$=>35$ & 2,917 & 85.2 & 1 & 30.9 & 1 & 11.0 & 1 \\
\hline $20-34$ & 9,356 & 89.7 & $1.20(0.90-1.54)$ & 34.7 & $1.23(0.82-2.31)$ & 13.9 & $1.11(0.52-1.93)$ \\
\hline$<20$ & 1,544 & 87.4 & $1.35(0.98-1.73)$ & 35.8 & $1.28(0.92-2.52)$ & 13.4 & $1.02(0.48-1.68)$ \\
\hline \multicolumn{8}{|c|}{ Birth order } \\
\hline $6+$ & 3,213 & 85.7 & 1 & 23.0 & 1 & 10.7 & 1 \\
\hline $4-5$ & 3,292 & 87.7 & $1.02(0.82-1.62)$ & 29.3 & $1.12(0.87-1.98)$ & 12.6 & $1.12(0.82-2.61)$ \\
\hline $2-3$ & 4,758 & 89.8 & $1.18(0.95-1.73)$ & 35.8 & $1.67(1.23-2.05)$ & 14.6 & $1.34(0.96-2.85)$ \\
\hline 1 & 2,554 & 90.4 & $1.22(0.86-1.68)$ & 48.5 & $2.31(1.98-2.86)$ & 15.0 & $1.43(1.23-2.97)$ \\
\hline \multicolumn{8}{|c|}{ Residence } \\
\hline Rural & 9,369 & 84.2 & 1 & 16.9 & 1 & 9.3 & 1 \\
\hline Asmara & 1,640 & 97.4 & $1.75(1.50-2.20)$ & 93.1 & $7.15(5.1-10.62)$ & 22.3 & $2.82(2.34-6.92)$ \\
\hline Other urban & 2,808 & 97.3 & $1.65(1.35-1.98)$ & 63.3 & $4.23(2.51-6.51)$ & 21.2 & $2.41(2.12-6.34)$ \\
\hline \multicolumn{8}{|c|}{ Administrative region (Zoba) } \\
\hline DebubawiKeihBahri & 209 & 61.2 & 1 & 34.3 & 1 & 13.5 & 1 \\
\hline Maekel & 2,533 & 95.6 & $1.38(1.24-1.50)$ & 73.6 & $2.67(2.39-2.78)$ & 22.7 & $1.96(1.61-2.24)$ \\
\hline SemenawiKeihBahri & 1,632 & 84.9 & $1.26(1.18-1.34)$ & 30.3 & $0.89(0.54-1.11)$ & 17.6 & $1.32(1.22-1.39)$ \\
\hline Anseba & 2,133 & 92.0 & $1.30(1.22-1.39)$ & 25.2 & $0.68(0.48-0.76)$ & 9.5 & $0.63(0.52-0.83)$ \\
\hline Gash-Barka & 3,445 & 84.7 & $1.22(1.96-2.35)$ & 17.8 & $0.52(0.35-0.74)$ & 8.6 & $0.78(0.70-0.98)$ \\
\hline Debub & 3,864 & 88.2 & $1.28(1.05-1.46)$ & 31.7 & $0.92(0.77-1.08)$ & 11.4 & $0.58(0.43-0.78)$ \\
\hline \multicolumn{8}{|c|}{ Mother's education } \\
\hline No education & 6,234 & 80.8 & 1 & 16.1 & 1 & 8.4 & 1 \\
\hline Primary & 3,429 & 92.7 & $1.50(0.96-1.85)$ & 29.9 & $2.32(2.47-2.97)$ & 13.4 & $1.52(1.39-1.71)$ \\
\hline Middle & 2,295 & 95.5 & $2.35(1.93-2.92)$ & 55.8 & $4.35(3.69-4.59)$ & 19.1 & $2.43(2.11-3.12)$ \\
\hline Secondary or above & 1,856 & 97.5 & $3.05(2.67-4.03)$ & 80.5 & $5.94(5.32-6.52)$ & 22.9 & $3.12(2.74-3.41)$ \\
\hline \multicolumn{8}{|c|}{ Current marital status } \\
\hline Never married & 2,878 & 77.5 & 1 & 39.5 & 1 & 25.3 & 1 \\
\hline Married/Living together & 6,183 & 89.6 & $2.62(1.52-4.35)$ & 65.8 & $1.33(0.57-1.93)$ & 46.7 & $1.20(0.64-2.52)$ \\
\hline
\end{tabular}


Journal of Complementary Medicine \& Alternative Healthcare

\begin{tabular}{|c|c|c|c|c|c|c|c|}
\hline Divorced/separated/ & & & & & \\
\hline Widowed & 1,177 & 72.8 & $1.21(0.96-1.53)$ & 42.5 & $1.12(0.42-1.78)$ & 19.8 & $1.11(0.53-1.92)$ \\
\hline \multicolumn{7}{|c|}{ Work status } \\
\hline Not employed currently & 7,883 & 85.6 & 1 & & \multicolumn{7}{c|}{1} & 1 \\
\hline Currently employed & 2,354 & 89.5 & $1.45(0.83-1.92)$ & & $1.1(0.72-1.85)$ & 21.8 & $0.85(0.57-1.33)$ \\
\hline \multicolumn{7}{|c|}{ Number of living children } \\
\hline 1
\end{tabular}

Results of the multivariate analysis showed that maternal age, birth order, residence, household socio-economic status and mother's education are related to the use of antenatal care (Table 2). Older women have very slightly lower antenatal care coverage (85\%) than younger women (87\%). Differences by birth order are in the direction one

Would expect but are very small: $90 \%$ for the first order, and $86 \%$ for $6+$ birth order [45]. There is a large difference of antenatal care coverage among urban (97\%) and rural (84\%) women. The proportion of antenatal care provided by doctors differs among urban $(21 \%)$ and rural women $(6 \%)$, urban seeing more than three times the coverage than in rural areas.

The results of the multivariate analysis in (Table 2) further showed that antenatal coverage is highest among the most educated women and those in the highest wealth quintile. For example, $81 \%$ of mothers with no education, as compared to $97 \%$ of women with secondary education utilize antenatal care. Women from richest quintile had 4.07 times more likelihood of receiving ANC during pregnancy in comparison with women from the poorest quintile of the society. The odds of reporting use of ANC by women with higher secondary and above education were 3.05 times higher than that of women with no education and women with primary education are $50 \%$ more likely than women with no education to receive antenatal care from a health professional.

Results of the multivariate analysis for the country as a whole reinforce the importance of place of residence and maternal age as second most important determinants of antenatal care utilization. Women residing in Asmara and in other urban areas are $75 \%$ and $65 \%$ more likely, respectively, than women in rural areas to receive antenatal care from a health professional. Urban residence had considerable positive association with the use of ANC. Other predictors of the use of antenatal care were mother's age at last birth, marital status, mother's occupation, and birth order. Women who were younger than 35 years at the time of last birth were 1.20 times more likely to receive ANC than women in the age group of 35 years or more. Married women are $62 \%$ more likely to receive antenatal care from a health professional than unmarried women. Regarding parity, the present study revealed that women with 2-4 children ever born were twice and half more likely to use antenatal care than women with only one child. In contrast, although high parity women (parity 5 or higher) tend to use the service more often than parity one women, the difference did not reach statistical significance. The birth order shows also slight difference in the utilization of antenatal care. Women with the 1st birth order are $20 \%$ more likely to use ANC and women on the 2-3 birth order are $18 \%$ more likely to use ANC than $6+$ birth order women. Employment has a positive influence on the use of ANC. Employed women are $45 \%$ more likely to use ANC.

Delivery care: The objective of providing safe delivery service is to protect the life and health of the mother and the baby. An important component of efforts to reduce the health risk to mothers and children is to increase the proportion of babies delivered under the supervision of health professionals. In Eritrea there is substantial variation in the use of delivery care services by residence, education, mother's age, birth order, administrative region and wealth quintile. The results of the multivariate analysis for the overall sample show that place of residence, women's education and number of children under five years old are independent predictors of utilization of delivery care services in Eritrea. The extent of variation in the use of delivery care services by residence is striking. Among those who reported having delivered their last child in a health facility, including the private sector, $73 \%$ were urban residents, $81 \%$ had secondary or above education and $90 \%$ were in the highest wealth quintile. Women residing in Asmara are about 7 times more likely to receive assistance during delivery than rural women, while women from other urban areas are about 4 times more likely to receive assistance during delivery than rural women. Women's education is also found to be an independent predictor of utilization of delivery care services in Eritrea, with the highest odds of utilization documented among women with secondary education and above (six times higher), middle level education (four times higher) and women with primary education (two times higher), compared with women with no education. 
(Table 2) also shows that professionally assisted deliveries to mothers are inversely related to women's parity. The likelihood of delivering in a health facility decreases with increasing birth order. Forty-eight percent of first order births delivered in health facilities, as compared to only $23 \%$ of the $6+$ birth orders (see EPHS 2010). Women with one child are 2.31 times more likely to receive professional delivery care than parity six and above women. It is generally believed that care during delivery would be higher for first order births and is expected to decline as order of birth increases [22,24]. Another interesting finding is that women with two or more children under five are $26 \%$ less likely to receive professionally assisted delivery services than women with only one child under age five. The extent of delivery care service utilization in Eritrea is striking, with $88.5 \%$ of pregnant women now receiving antenatal care from a health professional, only $34 \%$ delivered in health facilities with the assistance of trained health workers [45].

Over the last 15 years (1995-2010) the utilization of professionally assisted delivery care in health facility, in Eritrea, has increased by $17 \%$ only. There are several reasons for not delivering in a health facility. In the 2010 EPHS, women were asked whether each of the following factors would be a big problem or not a big problem in seeking medical care: getting permission to go for treatment, getting money for treatment, distance to a health facility, having to take transportation, not wanting to go alone, concern that there may not be a female health provider, and concern that there may not be a health provider available. The most common reasons given for not utilizing delivery services by Eritrean women was a concern that the facility was too far or not available (40\%), it was not necessary to deliver at a health facility (32\%) , and it is not being customary to deliver in a health facility (19\%). Looking at the regional variation for the reason for not utilizing delivery services, the survey revealed that $88 \%$ of Debubaw iKeih Bahri, $61 \%$ of Semenawi Keih Bahri \& Anseba women mentioned distance of health facility as a barrier, compared to $28 \%$ of women respondents from Maekel [45]. Most Gash-Barka respondents mentioned that it was not necessary to deliver in the health facility. Forty-eight percent of rural women mentioned distance as a barrier for service utilization, compared to $14 \%$ of urban women. Fifty-three percent of the lowest wealth quintile said distance as a barrier, compared to only $12 \%$ of the highest wealth quintile.

Postnatal care: This section presents findings relevant to child health and survival. It assesses vaccination and nutrition status of children, and treatment practices among children suffering from three childhood illnesses: acute respiratory infection, fever, and diarrhea. The EPHS 2010 shows that 83\% of children ages 12-23 months, in Eritrea, are fully vaccinated: 95\% had received the BCG vaccine, 93\% have received DPT 1-3 vaccinations, $91 \%$ had received polio $1-3$, and $91 \%$ had received the measles vaccine at any time before the survey. Two percent of children ages 12-23 months have not received any vaccinations. The coverage of the first DPT and polio vaccine is very high $(97 \%$ and $98 \%$, respectively). The immunization system was further strengthened with the introduction of pentavalent vaccine in 2008, as well as procurement of vaccines, availability of injection safety materials and training of service providers. These contributed to maintaining more than 80 per cent coverage of three doses of combined diphtheria/pertussis/tetanus vaccine, reducing vaccine-preventable diseases to the extent that no measles deaths or polio cases were reported, and the neonatal tetanus elimination target was achieved (see UNICEF 2012).Wide immunization coverage nation wise has resulted tremendous reduction in childhood mortality rates in Eritrea (Figure 1).

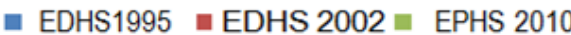

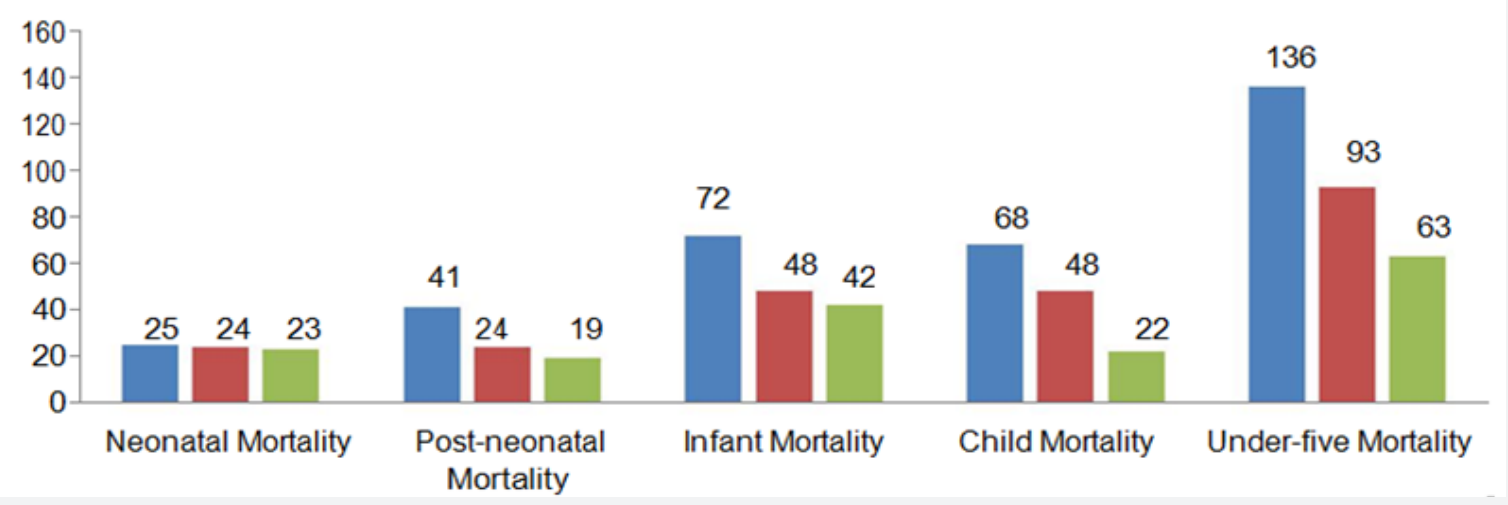

Figure 1: Trends in Childhood Mortality Rates.

As can be seen from Figure 1 under-five mortality rate has declined significantly by $32 \%$ from 93 deaths per 1000 live births in EDHS 2002 to 63 deaths per 1000 live births in EPHS 2010. The decline in child mortality is even more pronounced; from 48 deaths per 1000 children surviving age one in EDHS 2002 to 22 deaths in EPHS 2010, a decline of 54\%. Infant mortality has declined from 72 deaths per 1000 live births in EDHS 1995 to 42 deaths per 1000 live births in EPHS 2010. Despite huge improvements in child mortality the health status of many Eritrean children remains poor. Communicable and nutritional deficiency diseases such as diarrhea, acute respiratory infections, fever, malaria, skin and parasitic infections are still health 
problems in Eritrea. Acute Respiratory Infection (ARI) is one of the leading causes of child morbidity and mortality in Eritrea. According to the EPHS 2010 report $45 \%$ of children under the age of five with symptoms of ARI were taken to a health facility or provider for advice or treatment. Health-treatment- seeking behavior for children with ARI symptoms is more common among children age 6-11, 12-23 and 24-35 months (each with $50 \%$ ) but lowest in the age group 48-59 months (34\%) [45]. Urban children are also more likely than rural children to have been taken to a health facility or provider for treatment. Children of women with no education are less likely to be taken to a health facility or provider when they have ARI symptoms compared with children of mothers with secondary education or higher (38\% and 55\%, respectively).

The EPHS 2010 shows, overall, one-fifth of children under age five were reported to have had fever in the two weeks preceding the survey. The prevalence of fever varies with the age of the children. Generally the prevalence of fever peaked in the age group 6-11 and 12-23 months (30\% and 27\%, respectively) and then progressively decreased and is least in the age group less than 6 months (13\%). Children of mothers with no education (20\%) have the highest prevalence of fever when compared with their counter parts. The proportion of children with fever is higher in the middle and lower wealth quintiles (19-21\%) than in the highest wealth quintile (14\%). EPHS 2010 further shows, overall, $9 \%$ of all children under five had diarrhea, while $2 \%$ had diarrhea with blood. The occurrence of diarrhea varies by age of the child. Young children ages 6-23 months are more prone to diarrhea than children in the other group; those age 12-23 months have the highest prevalence $(17 \%)$ of diarrhea among the age cohorts. The prevalence of diarrhea decreases steadily with increasing wealth quintile and is lowest among children whose mothers have at least middle education. Overall, $34 \%$ of the children with diarrhea were taken for advice or treatment to a health facility or provider [45]. Advice or treatment for children with diarrhea is least sought for children whose mothers have no education (29\%) and high in secondary education or higher $(47 \%)$.

The study further shows that there is an inverse relationship between the length of the preceding birth interval and the proportion of children who are stunted. The long the interval, the less likely is that the child will be stunted. The mother's level of education general has an inverse relationship with stunting level. Children of mothers with secondary or higher education are the less likely to be stunted (31\%), while children whose mother's have no education are the most likely to be stunted (59\%). Children in the wealthiest households are also the least likely to be stunted (27\%). Children in rural areas are more likely to be stunted than in urban areas (56 versus 38 percent, respectively) [45].

The results of multivariate analysis showed that the stronger factor related to the use of PNC was skilled attendance at delivery (adjusted OR $=67.34 ; 95 \%$ CI 50.20-67.74). Further, the results showed that women from the richest households had higher likelihood of using postnatal care than women from the poorest households. The odds of reporting the use of postnatal care among women who were higher secondary and above educated were about 3.12 times higher than among those who were illiterate. Residence has also shows an influence on the utilization of postnatal care. Children living in urban areas are more likely than those living in rural areas to be fully vaccinated $(90 \%$ and $80 \%$, respectively). Women residing in Asmara are 2.82 times more likely to use PNC services than their rural counterparts. The corresponding odds ratio for women from other urban areas is 2.41.Likewise, association was found between the use of PNC and birth order and use of ANC during pregnancy. Vaccination coverage decreases as birth order increases; first births are more likely to be fully immunized (85\%) than births of order six and higher (79\%) [45].

\section{Discussion}

Most of the factors investigated are related to the demographic and socio cultural characteristics of women. The study has identified several factors that have important influence on utilization of maternal health services in Eritrea. These include mother's age, birth order, socio-economic status, place of residence, mother's education, and number of children under five. The multivariate analysis indicated that women delivering at younger age were more likely to use antenatal care, receive skilled attendance at delivery and use postnatal care. This study shows that among urban women, utilization of antenatal care is higher for those with two or more children than for those with only one child.

On the other hand, utilization of delivery care services is lower for those with two or more children than those with one child. While it is unclear why urban women who have just started childbearing are less likely to seek antenatal care than middle parity women, a possible explanation for the low utilization of delivery care services among high parity women is that such women developed confidence and may believe that modern health care is not as necessary due to the experience and knowledge accumulated from previous pregnancies and births. Also, most of the high parity women live in rural areas. With respect to the effect of parity on the utilization of delivery care, the results appear to be consistent with most studies done elsewhere, which indicate that women are significantly more likely to use delivery care services for their first child than later children [30,32,50-53]. One possible explanation for this is that women who are pregnant with their first child are usually more likely to have difficulties during labor and delivery than women of high parity. This may result in low parity women being more motivated to deliver in medical facilities than high parity women.

The findings further showed that the mothers used more antenatal care during the second order birth in comparison with the first order birth but the use of antenatal care decreased with birth order more than two. The levels of skilled attendance at delivery and postnatal care decreased steadily with increased 
birth order. The three indicators of the use of maternal health services increased sharply with increased levels of education of mother. All other variables used in this study were significantly associated with the use of the three maternal health services.

Household socio-economic status was the strongest factor associated with the use of the three maternal health service indicators. This analysis also indicated that women from families living at the lowest wealth quintile were less likely to use maternal health services in comparison with women living in families in the highest wealth quintile. The study further shows that coverage of delivery care and postnatal care services are very low in Eritrea as documented in EPHS 2010. Only about $34 \%$ percent and $13.3 \%$ of women received professionally assisted delivery and postnatal care services, respectively, in the five years preceding the survey. Coverage of services varies between regions, with DKB having the lowest coverage (42\%), and Gash Barka the highest (84.5\%).

HOs delivered only $14 \%$ of ANC services. Only $18.7 \%$ of pregnant women registered for ANC services in their first, 53.3\% in the second and $22 \%$ in the third trimester in 2006. The high drop out in the third trimester is said to be caused by lack of transport. Such levels of service coverage are considered low even by sub-Saharan standards. The results of Demographic and Health Surveys conducted in other sub-Saharan countries show coverage of delivery care utilization ranges from a low of $15 \%$ in Niger to 69 percent in Zimbabwe [48]. The lower coverage for delivery and postnatal care has often been attributable to the unpredictability in the onset of labor and the difficulty of travel, particularly for long distances, during labor, during delivery, and even within a few days after delivery. Moreover, the relatively high cost of delivery care is often blamed for the low rate of utilization of delivery services.

Mother's level of education was the second most influential factor for the use of modern maternal health services.Itis common predictor for the utilization of all the three maternity care services in Eritrea.On the national level, higher female education levels are associated with lower fertility rates, better nutritional status of children, a lesser prevalence of female genital mutilation, and increased use of family planning and AIDS prevention. Some of the results are impressive: women with secondary school education have fertility rates 44 percent lower than those with no education, their children are twice as likely to be well fed, and so on. Improvements in these indicators are even greater for women with secondary school education. Similar results were observed at the regional level: in regions with a large share of educated women (years of schooling), knowledge about HIV/AIDS is more common, family planning is used more widely, and children are better fed [45].

Mother's level of education is inversely related to her child's risk of dying. Although the relationship is not linear, children born to mothers with no education suffer the highest mortality at all ages. Brixiova et. al. [49] indicates that the infant mortality rate for children whose mothers have a primary education is 25 percent lower than that of children whose mothers have no education. The gap between children of mothers with at least a secondary education and children of mothers with no education is 36 percent. The corresponding figure for child mortality is 70 percent, and for under-five mortality, 51 percent. About 97 percent of women with at least secondary education know two or three ways of AIDS prevention, compared with 63 percent of women with no schooling. A woman with some secondary or higher education is twice as likely to receive antenatal care from a doctor as a woman without schooling [11]. The finding of a strong education effect is consistent with findings from elsewhere in the world $[28,31,32,50]$.

There are a number of explanations for why education is a key determinant of health service use. Education is likely to enhance female autonomy so that women develop greater confidence and capability to make decisions about their own health [51,52]. It is also likely that educated women seek out higher quality services and have greater ability to use healthcare inputs that offer better care [31].

Regional variations in access to health services are significant in Eritrea. Women living in the highlands, and especially in the cities, are more likely to visit health facilities, have their children vaccinated and send them to school than those living elsewhere. A higher proportion of women living in Asmara (Eritrea's capital city) receives antenatal care and gives birth at hospitals; and a higher number of children are monitored for growth and attend school than elsewhere. As a result maternal mortality and child malnutrition are lower in Asmara than in other places. In addition $40 \%$ of health professionals were assigned to Zoba Maekel in 2006. Thus, the reason for the high level of utilization of maternal health services among urban women compared with their rural counterparts is easily understood. As in most subSaharan countries, urban women in Eritrea tend to benefit from increased knowledge and access to maternal health services compared with their rural counterparts. This is because, health facilities are more accessible in urban areas and the various health promotion programs that use urban-focused mass media work to the advantage of urban residents and explain the close connection between urban residence and use of maternal health services. Moreover, rural women are more readily influenced by traditional practices that are contrary to modern health care.

Socio-cultural factors, which are difficult to model, are also appeared to influence the use of maternal and child health services. Cultural beliefs among the Tigre and Tigrigna ethnic groups, for instance, discourage delivery at health facility because they believe "it is unlucky for primigravidas to deliver at health facility" and "newly delivered mother and new born should not be exposed to the outside to protect them from "evil spirits" [44]. Women prefer home delivery even when they have access to health facilities. Traditionally childbirth is an event when the woman is surrounded and cared for by her family, friends and neighbors. 


\section{Journal of Complementary Medicine \& Alternative Healthcare}

Most women, in rural areas, prefer to deliver at home in familiar surroundings with the help of a traditional birth attendant (TBA) they know, trust and feel comfortable with. The gender of health staff (which is mostly male) is also a constraint for women who have inhibitions about being examined by a male health worker, especially in rural and in Moslem communities [44]. Of course, some women have overcome these inhibitions, but many more are staying away from essential medical checkups and thus endangering their health and that of their unborn baby. In the 2002 EDHS, about 37 percent of women (aged 15-49) in Zoba Debubawi Keih Bahri, 28 percent in Semenawi Keih Bahri, 20 percent in Anseba, and 26 percent in Gash-Barka, reported that concern there may not be of a female health worker is a big problem in accessing health care. In addition, many health staff assigned to rural health facilities is not from the ethnic group they serve and experience cultural and language barriers, which prohibits effective communication with their clients.

The health staff's general lack of interpersonal communication skills added with the cultural and language barriers become serious obstacles in the effort to promote health education and subsequent increases in health service utilization. There are a number of traditional medicinal practices that reflect the use of maternal and child health services in Eritrea. Eritrean traditional medicine is concerned not only with the curing of diseases but also with the protection and promotion of human physical, spiritual, social, mental and material wellbeing [54]. It deals with healing, prevention, as well as curative and surgical practices. The many categories of traditional medicinal practices dealing with these different aspects of health include: herbalists, bone setters, traditional psychiatrists, traditional pediatricians, traditional birth attendants (TBA), occult practitioners, herb sellers, spiritual healers, general practitioners, etc; they are certainly more readily available, accessible and approachable than the modern physicians while their services are much more affordable than modern medical facilities.

Traditional Eritrean medicine is commonly used to treat a variety of diseases employing substances as recommended by professional traditional medical practitioners. The conditions that claim to be treated include gastrointestinal disturbances, respiratory disorders, sexually transmitted infections, tuberculosis, impotency, hemorrhoids, rabies, intestinal parasites, skin problems, liver diseases, mental disorders, hypertension, diabetes, gynecological conditions, rheumatism, malaria, and surgical practices such as bone-setting, uvulectomy, circumcisions, bleeding and cupping, cautery, scarification and tooth extraction. About 85 percent of the respondents stated that they used traditional medicine to treat the aforementioned diseases.

In Eritrea, traditional medical treatments are also commonly given at the household level. There is significant knowledge of medicinal plants in the non professional public domain where most ailments are diagnosed and treated the household level. Where traditional professionals are consulted, it is often for their specialized traditional knowledge and skills pertaining to a relatively limited range of health. Most of the families grow or gather these plants in their vicinities of homes.

\section{Conclusion and Policy Implications}

Eritrea achieved half of the Millennium Development Goals, i.e. goals 4, 5, 6 and 72. The country has made significant progress in child and maternal health. The under-five mortality rate decreased from 136 per 1,000 live births in 1995 to 63 per 1,000 live births in 2010, and infant mortality declined from 72 per 1000 live births in 1995 to 42 per 1000 live births in 2010 . The maternal mortality declined from an estimated 1,400 per 100,000 live births in 1990 to 486 per 100,000 live births in 2010, and antenatal care increased from $49 \%$ in 1995 to $88.5 \%$ in 2010 [45]. The five major vaccine preventable diseases (poliomyelitis, measles, diphtheria, tetanus and whooping cough) no longer pose any major public health problem in Eritrea. The country has eliminated maternal and neonatal tetanus. Measles morbidity and mortality have been reduced to less than $90 \%$ of the 1991 levels. The country is heading towards achieving polio free status.

There have been tremendous improvements in early childhood mortality in Eritrea. Comparison of estimates of infant mortality from the EPHS 2010 and EDHS 2002 under-five mortality rate has declined by $32 \%$ from 93 deaths per 1000 live births in EDHS 2002 to 63 deaths per 1000 live births in EPHS 2010. The decline in child mortality is even more pronounced; from 48 deaths per 1000 children surviving age one in EDHS 2002 to 22 deaths in EPHS 2010. Infant mortality has declined from 72 deaths per 1000 live births in EDHS 1995 to 42 deaths per 1000 live.

Neonatal mortality in the most recent period $0-4$ years before the survey) is 23 deaths per 1000 live births. This rate is slightly higher than post neonatal deaths (19 per 1000 live births) during the same period; implying that Eritrean children who survived the first month of life have a lower risk of dying in the remaining 11 months of the first year of life. This indicates that $55 \%$ of infant deaths in Eritrea occur during the first month of life [45]. Two-thirds of the deaths under-five occur during the first year of life. This indicates that increased attention is required to the early postnatal period while maintaining existing satisfactory levels of immunization, community-based interventions and effective malaria and HIV control, as these have been instrumental in the rapid reduction of under-five mortality rates.

This study demonstrates that the utilization of maternal health care services is improved from 17\% (1995 EDHS) to 34\% (EPHS 2010). This is because, in order to increase facility based births, the MoH has established 15 Maternity Waiting Homes near Health Stations, where women can stay for up to 2 to 4 weeks before the expected delivery. The measure has shown to increase user rates. The ministry has also put in place maternal health promoters and peer educators to change the perceptions 


\section{Journal of Complementary Medicine \& Alternative Healthcare}

of communities about the services offered. TBAs also have been trained to identify complications and have been told to refer such patients.

This study shows that the most important factors influencing the use of maternal health services in Eritrea are demographic and socio cultural in nature. However, this does not detract from the relevance of service-related factors, especially in the rural areas. The demographic and socio cultural factors identified in this study include maternal education, place of residence, parity, and wealth quintile, which are similar to those documented in many settings throughout Africa and other developing countries. Such findings can therefore be used as the basis for a number of policy recommendations.

First, that education was found to have an important impact on the use of maternal health services suggests that improving educational opportunity for women may have a large impact on improving utilization of such services. This is, however, a longterm investment. During the last 23 years the social benefits of female education have been observed in Eritrea. The basic education and gender equality program produced good results, notably in the areas of access and equity to education. Successful approaches, such as the complementary elementary education and the nomadic education projects, were able to reach the very remote areas of the country and enroll 14,175 children ( 45 per cent girls) for the first time [47].The continuation of the adult literacy program (especially for women in rural and low-land areas) with some incentives (e.g. food for training); and inclusion of health education in the curricula will improve women's use of maternal and child health care services. There is a scope for accelerating progress in antenatal and postnatal care services.

These services have to be strengthened and coverage of emergency obstetric care expanded. Intensive sensitization and promotional campaigns need to be undertaken in order to raise community knowledge and attitude about the danger signs of pregnancy, delivery and postnatal as well as neonatal risks. Communities need to play a supportive role in emergency preparedness and readiness. The empowerment of women in making decisions regarding utilization of health services during pregnancy, childbirth and postpartum needs to be increased also. The Ministry of Health (MOH) should strengthen its information, education, and communication (IEC) programs and integrate them with adult literacy programs; and coordinate such programs with government and non-government organizations.

Second, that women at higher parity levels were found to be less likely to have deliveries assisted by modern professionals implies that parity should be one of the criteria for targeting education campaigns on the benefits of safe motherhood programs. Third, that rural woman were less likely to use the services means that maternal health care programs should be expanded and intensified in rural areas along with culturally appropriate education campaigns. Fourth, since women who are not married or in union are less likely to use the services, it is imperative to also target this group during education campaigns.
Fifth, the negative impact of traditional religion on the use of maternal health services points to the need for research into aspects of traditional religion that discourage the use of such health services.

Sixth, traditional medicine is commonly used for maternal and child health services in Eritrea beside modern health care services. Mothers use traditional medicine either because they are dissatisfied with modern medicine or because they seek self-control over their health care decisions of their kids. Traditional medical practitioners, diagnosing and managing various common diseases at PHC level, with various herbal dosage forms namely, concoctions, decoctions, infusions, dried powders, ointments, tinctures and macerates, are much closer to the community than modern medical practitioners who are mainly found in urban healthcare locations. Traditional medical practitioners administer these medications through various routes such as oral, rectal, intra-uterine, sub-cutaneous external or topical applications. In the traditional Eritrean society there is a holistic belief that the health of body, mind and spirit are related and that this should be taken into account by whoever cares for their health.

Seventh, herbal medicine has a huge potential in maternal and child health services in Eritrea. Eritrea has a rich tradition of plant use, an immense range of climates, cultures and species and has the human and natural resources to become an even greater producer of natural plant products. The pharmaceutical potentials of Eritrean medicinal plants are immense. In order to improve the situation of medicinal plants in Eritrea, a number of options come to mind. Urgent action is needed for research that focuses on the generation of baseline information on medicinal and aromatic plants and for promoting value-added processing of herbal medicines from local materials for local industries with simple dosage forms being standardized and packaged at low cost using appropriate technology. There are several official modern drugs today, which were originally developed like aspirin through traditional medicine e.g. morphine, digoxin, quinine, ergometrine, reserpine, atropine, etc and all of which are currently being used by orthodox medicine in modern hospitals all over the world [55].

Effective health agenda for Eritrean can never be achieved by modern biomedicine alone unless it is complemented by traditional medical practice. The most workable health agenda for Eritrea is the institutionalization of traditional medicine in parallel (not in complete fusion) with modern medicine, within the national health care scheme in order to move the health agenda forward. The Ministry of Health, therefore, should use such findings as an insight for the modification of plans and policies for future development of maternal and child care services in Eritrea.

\section{Acknowledgment}

The author extends his gratitude to the staff of Eritrean National Statistics Office. The entire study was based on the 


\section{Journal of Complementary Medicine \& Alternative Healthcare}

Eritrea Population and Health Survey of 2010 and without their support this study would be impossible.

\section{References}

1. EC/UNFPA (2000) Making pregnancy and child birth safer [Fact sheet] EC/UNFPA initiative for reproductive health in Asia in cooperation with the German foundation for world population. EC/UNFPA 2000.

2. World Bank (1994) Better health in Africa: experience and lessons learned. WorldBank, Washington D.C, USA

3. MOH (2012) Annual Health Services Activity Report 2012. Ministry of Health, Asmara, Eritrea.

4. UNDP (2009) UNDP Report.

5. Hadas Eritrea (2002) A note taken from Eritrean National Statistics Office "Population studies".

6. World Bank (2000) Reforming Public Institutions and strengthening Governance: The World Bank Strategy. Oxford, The World Bank, USA.

7. UNICEF (2004) The State of the World's Children.

8. World Bank (2002) World Development Report. Oxford University Press: World Bank, New York, USA.

9. World Bank (2002) Making Services Work for Poor People, World Development Report 2004. Oxford University Press, World Bank, New York, USA.

10. MOH (2003) Annual Health Services Activity Report (JanuaryDecember 2002). Ministry of Health Asmara, Eritrea.

11. EDHS (Eritrea Demographic and Health Survey) (2002) National Statistics and Evaluation Office (NSEO) [Eritrea] and ORC Macro, Calverton, Maryland, USA.

12. EDHS Eritrea Demographic and Health Survey (1995) National Statistics and Evaluation Office (NSEO)[Eritrea] and ORC Macro (1996), Calverton, Maryland, USA.

13. Fauveau V, M Koenig, J Chakraborty, A Chowdhury (1988) Causes of maternal mortality in rural Bangladesh: 1976-1985. Bulleting of the World Health Organization 66(5): 643-651.

14. Fortney J, I Susanti, S Gadalla, S Saleh, P Feldblum, et al. (1988) Maternal mortality in Indonesia and Egypt. British Journal of Gynecology and Obtetics 26(1): 21-32.

15. Fiedler J (1981) A review of the literature on access and utilization of medical care with special emphasis on rural primary care. Social Science and Medicine 15(3): 129-142.

16. Kroeger A (1983) Anthropological and socio-medical health care research in developing countries. Social Science and Medicine 17(3): 147-161.

17. Rosenstock I (1966) Why people use health services. Milbank Memorial Fund Quarterly 44(3): 94-124.

18. Anderson R, Newman J (1973) Social and individual determinants of medical care utilization in the United States. Milbank Memorial Quarterly 51(1): 95-124.

19. Newbold, K Bruce (1995) Equity in health care: Methodological contribution to the analysis of hospital utilization within Canada. "Social Science and Medicine 40(9): 1181-1192.

20. Henderson, Gail (1994) Equity and Utilization of Health services: Report of an eight-province survey in China. Social Science and Medicine 39(5): 687-699.

21. Fosu G (1994) Childhood mortality and health service utilization: cross-national comparisons of user-related factors from DHS data. Social Science and Medicine 38: 1209-1220.
22. Bhatia J, Cleland J (1995) Determinants of maternal care in a region of South India. Health Transition Review 5: 127-142.

23. Addai I (2000) Determinants of use of maternal-child health services in rural Ghana. Journal of Biosocial Science 32(1): 1-15.

24. Elo T (1992) Utilization of maternal health services in Peru: The role of women's education. Health Transition Review 2: 49-69.

25. Wong E, Popkin B, Gullkey D, Akin J (1987) Accessibility, quality of care \& prenatal care use in the Philippines. Social Science and Medicine 24: 927-944.

26. McKinlay J (1972) Some approaches and problems in the study and use of services: an overview. J Health and Soc Behav 13(2): 115-152.

27. Kwast B, J Liff (1988) Factors associated with maternal mortality in Addis Ababa, Ethiopia. International Journal of Epidemiology 17(1): 115-121.

28. Becker S, Peters D, Gray R, Gultiano C, Black R (1993) The determinants of use of maternal and child health services in Metro Cebu, The Philippines. Health Transition Review 3: 77-89.

29. Costello M, Lleno L, Jensen E (1996) Determinants of two major earlychildhood disease and their treatment in the Philippines. Findings from the 1993 National Demographic Survey. Asia Pac Popul Res Abstr (9): $1-2$.

30. Leslie J, G Gupta (1989) Utilization of formal services for maternal nutrition and health care. Washington, D.C. USA.

31. Celik Y, D Hotchkiss (2000) The socioeconomic determinants of maternal health care utilization in Turkey. Social Science \& Medicine 50(12): 1797-1806.

32. Stewart K, A Sommerfelt (1991) Utilization of maternity care services: A comparative study using DHS data. Proceedings of the Demographic and Health Surveys World Conference, Washington, DC 3: 1645-1668.

33. Pelto G (1987) Cultural issues in maternal and child health and nutrition. Social Science \& Medicine 25(6): 553-559.

34. Adetunji J (1991) Response of parents to five killer diseases among children in a Yoruba community, Nigeria. Social Science \& Medicine 32(12): 1379-1387.

35. WHO (1998) Improved access to maternal health services. WHO, Geneva, Switzerland.

36. Abbas A, Walker G (1986) Determinants of the utilization of maternal and child health services in Jordan. International Journal of Epidemiology 15: 404-407.

37. Stock R (1983) Distance and utilization health facilities in rural Nigeria. Social Science and Medicine 17: 63-70.

38. Airey T (1998) The impact of road construction on hospital in-patient catchment in the Meru district of Kenya. Social Science and Medicine 29(1): 95-105.

39. Paul B (1991) Health service resources as determinants of infant death in rural Bangladesh: an empirical study. Social Science and Medicine 32: $43-49$

40. Williams C, N Baumslag, D Jelliffe (1985) Mother and child health: Delivering the services. ( $2^{\text {nd }} E d n$.), London: Oxford University Press.

41. Bicego G, Siân C, Hendrik R, Saidi K, Sylvester N (1997) Sumve Survey on Adult and Childhood Mortality, Tanzania, 1995: In-depth study on estimating adult and childhood mortality in settings of high adult mortality. Macro International Inc., Calverton, Maryland.

42. Gertler P, J van der Gaag (1988) Measuring the willingness to pay for services in developing countries. LSMS working paper No. 45. The World Bank, Washington, D.C., USA. 
43. Gertler P, L Locay, W Sanderson (1998) Are user fees regressive? The welfare implications of health care financing proposals in Peru. In: P Gertler \& L Locay (Eds.), Health care financing and the demand for medical care, LSMS working paper No. 37. The World Bank, Washington, D.C., USA.

44. Habtom G, Ruys P (2007) The Choice of a Health care Provider in Eritrea. Health Policy 80(1): 202-217.

45. EPHS Eritrea Population and Health Survey (2010) National statistics Office and Fafo Institute for Applied international Studies.

46. Rutstein S, Johnson K, Gwatkin D (2000) Poverty, Health Inequality, and Its Health and Demographic Effects. Paper Presented at the 2000 Annual Meeting of the Population Association of America, Los Angeles, California.

47. UNICEF (2012) Eritrea Country Program 2013-2016. Draft Country Program Document (CPD) for Eritrea (E/ICEF/2013/P/L/1).

48. Stewart M, Stanton C, Ahmed O (1997) Maternal health care. DHS Comparative Studies No. 25. Macro International Inc., Calverton, Maryland.

49. Brixiova Z, Ales B, Joshua C (2001) The gender gap in education in Eritrea in 1991-1998: A missed opportunity? IMF Working Paper.

This work is licensed under Creative Commons Attribution 4.0 Licens

DOI:10.19080/JCMAH.2017.02.555589
50. Akin A, Munevver B (1996) Contraception, abortion and maternal health services in Turkey: Results of further analysis of the 1993 Turkish Demographic and Health Survey. Ministry of Health [Turkey] and Macro International Inc., Calverton, Maryland.

51. Caldwell J (1981) Maternal education as a factor in child mortality. World Health Forum 2(3-4): 75-78.

52. Raghupathy (1996) Education and the use of maternal health care in Thailand. Social Science \& Medicine 43(4): 459-471.

53. Adekunle C, Filippi V, Graham W, Onyemunwa P, Udjo E (1990) Patterns of maternitycare among women in Ondo States, Nigeria. In: Allan G. Hill, Determinants of health and mortality in Africa. Demographic and Health Survey Further Analysis Series No. 10, 1-45. The Population Council, New York, USA.

54. Habtom G (2015) Integrating traditional Medical Practice with Primary Healthcare System in Eritrea. Journal of Complementary and Integrative Medicine 12(1): 71-78.

55. Elujoba A, Odeleye O, Ogunyemi C (2005) Traditional Medicine Development for Medical and Dental Primary Healthcare Delivery System in Africa. Afr J Trad CAM 2(1): 46-61.

\section{Your next submission with Juniper Publishers will reach you the below assets}

- Quality Editorial service

- Swift Peer Review

- Reprints availability

- E-prints Service

- Manuscript Podcast for convenient understanding

- Global attainment for your research

- Manuscript accessibility in different formats

( Pdf, E-pub, Full Text, Audio)

- Unceasing customer service

Track the below URL for one-step submission https://juniperpublishers.com/online-submission.php 